\title{
Herpes simplex viruses: is a vaccine tenable?
}

\author{
Richard J. Whitley ${ }^{1}$ and Bernard Roizman ${ }^{2}$ \\ ${ }^{1}$ Department of Pediatrics, Microbiology and Medicine, University of Alabama at Birmingham, Birmingham, Alabama, USA \\ ${ }^{2}$ The Marjorie B. Kovler Viral Oncology Laboratories, The University of Chicago, Chicago, Illinois, USA \\ Address correspondence to: Richard J. Whitley, University of Alabama at Birmingham Children's Hospital, ACC 616, \\ 1600 Seventh Avenue South, Birmingham, Alabama 35233, USA. \\ Phone: (205) 934-5316; Fax: (205) 934-8559; E-mail: rwhitley@peds.uab.edu. \\ J. Clin. Invest. 109:145-151 (2002). doi:10.1172/JCI200216126.
}

Human herpes simplex virus (HSV) infections have been documented since ancient Greek times. Greek scholars, notably Hippocrates, used the word "herpes," meaning to creep or crawl, to describe spreading cutaneous lesions. Over the ensuing centuries, the clinical manifestations were described, including a distinction between genital and orofacial herpes, accounting for the designation of HSV-1 and HSV-2. Importantly, these viruses have a unique propensity to establish latency and recur over time, in spite of specific host immune responses, making successful vaccine development a challenge. The spectrum of disease caused by HSV includes primary and recurrent infections of mucous membranes (e.g., gingivostomatitis, herpes labialis, and genital HSV infections), keratoconjunctivitis, neonatal HSV infection, visceral HSV infections of the immunocompromised host, encephalitis, Kaposi varicella-like eruption, and an association with erythema multiforme. As it relates to genital herpes, $22 \%$ of the general population is infected with HSV-2, and the seroprevalence is even higher: $30-50 \%$ in patients attending sexually transmitted diseases clinics. Here, we will focus on HSV vaccine development with specific reference to translational molecular biology.

Molecular virology relevant to vaccine development A brief discussion of HSV's molecular biology will set the stage for a discussion of the current status of vaccines. HSVs are members of a family of viruses whose genomes consist of a single large double-stranded DNA molecule (1). The HSV virion consists of four components: (a) an electron-dense core containing viral DNA; (b) an icosadeltahedral capsid; (c) an amorphous, attimes eccentric layer of proteins, designated tegument, which surrounds the capsid; and (d) an envelope. The capsid consists of 162 capsomeres and is surrounded by the tightly adhering tegument. The envelope surrounds the capsid-tegument structure and consists of at least 11 glycosylated and several nonglycosylated viral proteins, lipids, and polyamines. Host immune responses are greatest against the structural proteins and the envelope glycoproteins. The DNA of HSV-1 and -2 consists of two covalently linked components, designated L (long) and S (short). Each component is composed of unique sequences (UL or US, respectively) flanked by relatively large inverted repeats. The inverted repeat sequences flanking UL are $a b$ and $b^{\prime} a^{\prime}$, whereas those flanking US are $a^{\prime} c^{\prime}$ and $c a$. The two components can invert relative to one another to yield four populations of DNA molecules differing solely in the relative orientation of these DNA sequences (2). Knowledge of the structure of HSV allowed for the development of genetic engineering to generate candidate vaccines and gene therapy vectors.

HSV-1 - and, by extension, HSV-2 - are now thought to encode at least 84 different polypeptides (3). To initiate infection, HSV must attach to cell-surface receptors, fuse its envelope to the plasma membrane, and allow the de-enveloped capsid to be transported to the nuclear pores, where DNA is released into the nucleus. The key events in viral replication that occur in the nucleus include transcription, DNA synthesis, capsid assembly, DNA packaging, and envelopment. Viral surface glycoproteins, key targets of protective humoral responses, mediate attachment and penetration of the virus into cells. Currently at least 11 viral glycoproteins (designated $\mathrm{gB}, \mathrm{gC}, \mathrm{gD}, \mathrm{gE}, \mathrm{gG}, \mathrm{gH}$, $\mathrm{gI}, \mathrm{gJ}$, gK, $\mathrm{gL}$, and $\mathrm{gM}$ ) are known, and another is (gN) predicted. Two of the glycoproteins encoded by HSV-2, gB-2 and gD-2, have been tested in subunit vaccines, and a mutant lacking $\mathrm{gH}$ is a candidate for use as a disabled infectious single cycle (DISC) vaccine (4).

The synthesis of viral gene products, both RNA and proteins, takes place in three sequential waves. The $\alpha$, or immediate early, proteins regulate the reproductive cycle of the virus or block antigenic peptide presentation on the infected cell surfaces. The $\beta$, or early, proteins, synthesized next, are responsible for viral nucleic acid metabolism and are the main target of antiviral chemotherapy. These gene products include the viral thymidine kinase and the viral DNA polymerase. Finally, the late, or $\gamma$, gene products are, for the most part, the structural components of the virion (1).

Several aspects of HSV protein function are relevant to the pathogenesis of human disease, and to prospects for prophylaxis and antiviral chemotherapy. First, most 
viral proteins examined to date play multiple roles and, in many instances, interact with diverse cellular proteins. They are also extensively modified posttranslationally, and evidence is mounting that the specific function expressed by each protein is determined at least in part by their modifications. Second, of the 84 genes, as many as 47 can be deleted singly or in small groups without affecting the ability of the virus to replicate - at least in some cultured cell types.

Most of these genes, although dispensable in vitro, are essential for efficient replication in experimental animal systems. Many of the dispensable viral proteins, as well as some of the essential ones, are designed to scavenge cells for useful cellular proteins capable of blocking any attempt of the host to inhibit viral replication. Thus, a tegument protein, designated virion host shutoff (vhs), induces degradation of cellular and viral RNA early in infection. The sheer volume of viral RNA transcripts accumulating in infected cells enables viral gene expression. The infected cell protein 27 (ICP27), which is made immediately after infection, is another example. This protein blocks splicing of cellular mRNA, thereby reinforcing the shutoff of cellular protein synthesis initiated by vhs. In addition, since viral entry into cells and expression of viral gene functions induce a stress response that can, if unchecked, activate proapoptotic pathways that modulate virus spread. At least three different viral proteins, glycoproteins $\mathrm{D}$ and $\mathrm{J}$ and the protein kinase US3, play a role in blocking programmed cell death induced by exogenous agents or by viral gene products.

Another viral gambit that nullifies a protective host response involves the HSV protein $\gamma_{1} 34.5$. Since open reading frames exist on both strands of HSV DNA, complementary transcripts abound in the infected cell, leading to activation of protein kinase R (PKR), phosphorylation of the $\alpha$ subunit of the translation initiation factor eIF2, and total shutoff of protein synthesis. Activation of PKR is an innate response to infection; most viruses have evolved one or multiple mechanisms to evade it. Protein $\gamma_{1} 34.5$ binds protein phosphatase 1 and redirects it to dephosphorylate eIF $2 \alpha$. The dephosphorylation of eIF $2 \alpha$ is highly efficient and insures uninterrupted synthesis of viral proteins. Viral protein ICP47, made immediately after infection, blocks immune responses to HSV-infected cells by binding the antigenic peptide transporter TAP1/TAP2, thus preventing the presentation of viral epitopes on MHC class I proteins of infected cells. Interestingly, two viral products, vhs and $\gamma_{1} 34.5$, cooperate to block endocytosis and the transport of $\mathrm{MHC}$ class II to the cell surface. ICP0 has recently been shown to function as a ubiquitin ligase, which is now thought to specifically target for degradation proteins that are inimical to viral replication.

Finally, certain gene products that are important in vivo but dispensable in cell culture help ensure the proper cellular environment and mobilization of cellular resources necessary for efficient viral replication. Key proteins of this class may be found in the tegument or expressed among the $\alpha$ genes. These include the $\alpha$-trans-inducing factor $(\alpha$ TIF), or VP16, which augments the basal level of expression of $\alpha$ genes; ICP4, which both promotes and represses viral gene expression; ICP22, which controls the expression of a subset of late genes; and ICP27, which shuttles RNA from nucleus to cytoplasm late in infection.

Thus, the immunity induced by an HSV vaccine must overcome the highly evolved capacity of the virus to hide the infected cell from the immune system and the very efficient machinery to produce virus progeny, allowing it to spread from cell to cell.

\section{HSV involvement of neurologic tissues}

Two unique biologic properties of HSVs profoundly influence human disease and will need to be targeted by a successful vaccine. First, these viruses can invade and replicate in the CNS. Deletion of virtually any of the genes dispensable for viral replication in cell culture reduces the capacity of the virus to invade and replicate in CNS. Mutations affecting neuroinvasiveness have also been mapped to glycoprotein genes. Since neuronal cells do not make cellular DNA, they lack the precursors for viral DNA synthesis that are also encoded by the viral genes dispensable for replication in cell culture. $\gamma_{1} 34.5$ is of particular interest in that mutants deleted in this gene are among the least virulent known, even though they multiply well in a variety of cells in culture (5). The molecular basis for the failure of $\gamma_{1} 34.5$ mutants to multiply in the CNS is unclear and may not be related to the protein's known ability to dephosphorylate eIF $2 \alpha$.

The second salient characteristic of the HSVs is that of latency. Following entry into and infection of nerve endings, both HSV-1 and HSV-2 are transported in a retrograde fashion to the nuclei of sensory ganglia, where they multiply in a small number of sensory neurons. In the vast majority of the infected neurons, the viral genome remains in an episomal state for the entire life of the individual. Reactivations can occur in spite of ongoing cell-mediated and humoral immune responses and may be induced by a variety of local or systemic stimuli, such as physical or emotional stress, fever, exposure to ultraviolet light, or tissue damage, as well as by immune suppression. Recurrent herpes labialis is three times more frequent in febrile patients than in nonfebrile controls. Latent virus can be retrieved from the trigeminal, sacral, and vagal ganglia of humans either unilaterally or bilaterally (6). The recovery of virus by in vitro cultivation of trigeminal ganglia helps explain the observation of vesicles that recur at the same site in humans, usually the vermilion border of the lip.

Little is known regarding the mechanisms by which the virus establishes and maintains a latent state or becomes reactivated. Likely, establishment of latency is based on several events occurring simultaneously. These could well involve the expression of viral genes that block proteins from being made, the absence of factors that enable high-level expression of genes, or the induction of cellular factors that repress viral gene expression. 


\section{The rationale for HSV vaccines}

An efficacious HSV vaccine is very much needed, as indicated best by disease burden. In the US alone, over 100 million individuals are infected by HSV-1, and at least 40 million to 60 million individuals have been infected by HSV-2. Annually, a minimum of 2500 cases of neonatal herpes and 3000 cases of herpes simplex encephalitis result in significant morbidity and mortality in spite of efficacious antiviral therapy. Furthermore, because HSV results in genital ulcerative disease, the risk of acquisition of $\mathrm{HIV}$ is significantly increased by a factor of three or more.

An ideal vaccine should induce immune responses adequate to prevent infection. If primary infection were prevented, the colonization of the sensory ganglia would not occur and, therefore, no source of virus for either subsequent recurrences or transmission would exist. No one knows whether these objectives can be met. Short of this ideal, a candidate vaccine might be considered successful if it (a) mitigates primary clinical episodes, (b) prevents colonization of the ganglia, (c) helps reduce the frequency or severity of recurrences, and (d) reduces viral shedding in actively infected or asymptomatic individuals. These goals must be approached in light of the age of the target population and the duration of the desired results. Arguably, fundamental to a successful vaccine is its ability to prevent person-to-person transmission. Prospective clinical trials, a time-consuming and expensive exercise, will be required to appropriately define the true utility of an HSV vaccine using appropriate markers of clinical efficacy. Toward this end, it is of the utmost importance to determine which aspects of the host response - humoral or cell-mediated immunity; local or systemic immunity; antibodydependent or -independent cellular cytotoxicity - are protective against HSV infection (7).

Based upon human epidemiologic data, the rationale for an HSV vaccine is fourfold. First, exogenous reinfection is exceedingly uncommon in the immunecompetent host (6). Second, many more individuals are infected by HSV than either shed viruses or experience recurrences. Third, prior HSV-1 infection ameliorates the frequency and severity of subsequent HSV-2 disease. Finally, transplacental antibodies significantly decrease the risk of infection in the exposed newborn at the time of delivery. Additional encouraging findings come from experiments in animal models. Thus, both humoral and cell-mediated immune responses have been effective in a variety of animal species in preventing HSV challenge. Passive antibody administration with either polyclonal or monoclonal antibodies specific for $\mathrm{gB}-2$ and/or $\mathrm{gD}-2$ protects against experimental challenge in murine and guinea pig models. Immunization with either or both of these glycoproteins results in immune responses that prevent viral disease. Lastly, the genetic engineering of HSV has led to replication-competent and DISC vaccines that are similarly efficacious in several animal models. Taken together, these observations strongly suggest that an efficacious vaccine could be designed.

\section{HSV vaccine development}

Two of the more promising approaches to HSV vaccine development now being pursued build upon entirely different theoretical approaches. The first is based on the use of $\mathrm{gB}-2$ and/or $\mathrm{gD}-2$ as subunit vaccines in combination with an adjuvant. The second is a genetically engineered live, attenuated vaccine from which putative neurovirulence sequences have been removed. However, prior attempts at HSV vaccine development offer important lessons for the design of clinical and preclinical studies to evaluate vaccines of this kind.

\section{Live and inactivated wild-type virus vaccines}

Numerous clinicians attempted to alter the pattern of recurrences by inoculation of autologous virus, of virus from another infected individual, or, in one set of experiments, of virus recovered from an experimentally infected rabbit. The consequences were obvious, with lesions appearing at the site of inoculation in $40-80 \%$ of volunteers. In spite of the appearance of lesions, the evaluation of only a very limited number of patients, and the absence of important controls, efficacy was reported. In some cases, inoculation led to recurrences (8). Such live-virus vaccines were abandoned on the grounds that many patients did not develop lesions at the site of inoculation and, therefore, it was not perceived that the patient had an "adequate take."

Killed virus vaccines have been studied in a variety of animal models, often with good results. Unfortunately, most studies of HSV-infected individuals receiving these preparations failed to include an appropriate control group. Because patients have been reported to experience a $30-70 \%$ decrease in the frequency of recurrences as well as improvement in severity simply from having received placebo $(9,10)$, such controls are clearly needed to evaluate the protection afforded by the vaccine. The initial inactivated vaccines had the additional disadvantage that they were derived from phenol-treated infected animal tissues. Because of the possibility that administration of animal proteins might lead to demyelination, these vaccines did not attract much biomedical attention. More recently, ultraviolet light inactivation of purified virus derived from tissue culture has replaced phenol inactivation.

Over the past two decades, numerous studies have yielded conflicting evidence about the value of the killed-virus approach. Viral antigen obtained from amniotic or allantoic fluid, chorioallantoic membranes, chick cell cultures, sheep kidney cells, or rabbit kidney cells and inactivated by formalin, ultraviolet light, or heat led to a series of vaccine studies in thousands of patients $(9,11-13)$. Unfortunately, placebocontrolled studies of inactivated HSV vaccines are rare $(10,14)$, and the results have been widely discrepant, even between studies using the same vaccine. Few side effects are generally noted, although some authors have noted their concern that, in patients with keratitis, autoimmune phenomena might make the herpetic disease worse (14). With one exception, each of these studies reported significant improvement in as many as $60-80 \%$ of patients $(12,13)$. Interestingly, these studies 
show that, despite repeated inoculations, antibody titers (as measured by neutralization or complement fixation) remain little changed in most subjects. Some investigators have concluded that vaccination provided some initial benefit for patients with recurrent infection, but that long-term benefit could not be established. The only prospective study of prevention of HSV infections by vaccination was performed by Anderson et al. in children in an orphanage (15). In this study, ten children received vaccine and ten placebo; HSV stomatitis developed in an equal number of patients on follow-up.

\section{Subunit vaccines}

Subunit vaccines evolved out of attempts to remove viral DNA and eliminate the potential for cellular transformation, to enhance antigenic concentration in order to induce stronger immunity, and, finally, to exclude any possibility of residual live-virus contamination. The immunogenicity and efficacy of glycoprotein vaccines derived from the entire HSV envelope, free of viral DNA, have been demonstrated in animals (16-24). Neutralizing antibodies are detected in varying amounts, correlating with the degree of protection upon challenge. Because of the variety of challenge models and the use of different routes and dosages, interpretation of these results is extremely difficult. While there are conflicting animal model studies, in general, the subunit vaccines appear to elicit a degree of protection, as evidenced by amelioration of morbidity and reduction in mortality in the immunized animals. Nevertheless, several injections were required to induce protection, and adjuvant must be included as well. Protection of rodents is significantly more successful than that of higher primate species, perhaps reflecting the fact that HSV is not indigenous to rodent species. Such protection studies may therefore be totally irrelevant when evaluating human responses. Vaccination of primates, specifically rhesus monkeys, chimpanzees (17), and cebus monkeys, induces neutralizing antibodies, leading to an amnestic response following subsequent injection months later, but the significance of even these data for human experimentation remains unclear.

Several human subunit vaccine trials have now been completed. One of the earliest human vaccine experiments was with a Merck Sharp \& Dohme (Rahway, New Jersey, USA) glycoprotein envelope vaccine (25), which was produced from purified envelope glycoproteins. In a phase IIA study, carried out in sexual partners of patients known to have genital herpes, the number of individuals developing herpetic infection was nearly equal in placebo and vaccine recipients; thus, vaccination failed to provide any benefit at all. More recent clinical trials have evaluated the Chiron Corporation (Emeryville, California, USA) gB-2 and gD-2 and SmithKline Beecham (King of Prussia, Pennsylvania, USA) gD-2 purified subunit vaccines in humans. These vaccines incorporate one or both glycoproteins, as well as adjuvants unique to each company. This work provided important lessons for vaccine development: Extensive rodent experiments employing the guinea pig and murine genital herpes models demonstrated that either combined gB-2 and gD-2 or gD-2 alone, with Freund's adjuvant, completely protected against both primary and spontaneous recurrent disease following intravaginal viral inoculation (22). However, because complete Freund's adjuvant is not acceptable for human administration, alternative adjuvants have been explored, including Chiron's MF-59 and a proprietary SmithKline Beecham adjuvant. Both afford a high level of protection from HSV disease (26). The quantity of neutralizing antibody elicited by immunization and the total HSV antibody titers were higher after vaccination, and the latter value correlated with protection from disease $(22,27)$.

Data from the largest published series of vaccinated individuals with the Chiron construct failed to demonstrate significant long-term prevention of infection in susceptible sexual partners, although initial benefit was apparent for the first 5 months. In this trial, there was a $50 \%$ reduction in the rate of infection during this short window. The overall efficacy of the vaccine was $9 \%$, although the vaccine had no effect on the frequency of recurrences. Even when all variables that could have influenced acquisition of infection were controlled, the beneficial effect was maintained. This large multi-institutional trial was preceded by a carefully performed phase II study that suggested clinical benefit (28). No further vaccine studies are planned for this construct. Of note, the adjuvant for these studies was MF-59, a potent inducer of Th2 responses.

Another series of clinical trials on gD-2 from Glaxo SmithKline has been reported (29). Here, the adjuvant was alum plus monophosphoryl lipid A, a potent inducer of Th1 responses. In these studies, women who were seronegative for both HSV-1 and HSV-2 were protected from both disease ( $72 \%$ efficacy) and infection ( $43 \%$ efficacy). However, no significant clinical benefit could be demonstrated for individuals seropositive for HSV-1 (irrespective of sex) or for seronegative men. These results are being pursued in follow-up clinical trials.

\section{Live vaccines revisited}

Live vaccines, in general, are considered preferable to killed or subunit vaccines, because they are more likely to induce a broad range of immune responses to the expressed gene products, providing a higher level of protection, as with such viral pathogens as measles, mumps, and rubella. Furthermore, since these vaccines replicate in the recipient, the resulting immunity should be longer-lasting. Moreover, they usually require smaller doses of antigen and, therefore, should be more economical. In addition to the direct approach of using live HSV (typically an avirulent virus), it is now possible to engineer other viruses to display the HSV epitopes. Alternatively, HSV itself can be engineered to produce a vaccine strain that is protective without causing human disease. This latter approach is particularly attractive because nonpathogenic strains derived from serial passage in cell culture or animal hosts readily revert to pathogenicity. This lack of genetic stability is unacceptable for potential human vaccines (27).

Vaccinia virus has been proposed as a vector for delivering antigens to humans (30). The principle of insert- 
ing foreign genes into a vaccinia vector was exploited for the expression of the $g D$ and $g B$ genes of HSV (31-33). Unfortunately, vaccinia has been shown to cause vaccinia gangrenosum and disseminated vaccinia in individuals who were vaccinated to prevent smallpox. Moreover, immune memory in individuals who have previously received vaccinia may prevent recognition of any foreign gene insert. Adenoviruses and canary pox have also been proposed as alternative expression vectors, on the grounds that they might be safer than vaccinia (34).

Recombinant HSVs have also been constructed as prototypes of HSV vaccines, using a technology developed by Post, Roizman, and colleagues (35-37). These vaccines were designed to be attenuated, to protect against HSV-1 and HSV-2 infections, to provide serological markers of immunization different from those that would be seen following wild-type infections, and to serve as vectors to express immunogens of other human pathogens. To excise some of the genetic loci responsible for neurovirulence and to create convenient sites and space within the genome for insertion of other genes, the genome of HSV-1 strain HSV-1(F) was deleted in the domain of the viral thymidine kinase $(T K)$ gene and in the inverted repeats flanking the junction region between the UL and US sequences. An HSV-2 DNA fragment encoding the HSV-2 glycoproteins D, J, $\mathrm{G}$, and I was inserted in place of the internal inverted repeat. These type 2 genes were included in the hope of broadening the spectrum of the immune response and to create a chimeric pattern of antibody specificities as a serological marker of vaccination. The resulting recombinant, designated R7017, lacks TK activity and should therefore be resistant to acyclovir. Another recombinant, designated R7020, was generated by inserting the TK gene next to the HSV-2 DNA fragment. Since this virus expresses TK, it is susceptible to antiviral chemotherapy with acyclovir.

In rodent models, these two constructs induce protective immunity but appear considerably attenuated in their pathogenicity and ability to establish latency. The recombinants did not regain virulence, nor did they change DNA restriction enzyme cleavage patterns when subjected to serial passages in the mouse brain (37). Surprisingly, the TK- virus, R7017, was no more attenuated than the $\mathrm{TK}^{+}$virus, R7020. These results have been corroborated by studies in owl monkeys (38): While 100 plaque-forming units (PFU) of wild-type viruses administered by peripheral routes were fatal to the monkeys, recombinants given by various routes in amounts at least 105 -fold greater were innocuous or produced mild infections, even in the presence of immunosuppression by total lymphoid irradiation (37).

Unfortunately, human studies with this vaccine have been disappointing. The maximum dose of vaccine administered, $10^{5} \mathrm{PFU}$, elicited only mild immunogenicity, even with the administration of two doses (38). In many respects, the R7020 construct appears overly attenuated. However, as noted below, this virus is now being studied for gene therapy of adenocarcinoma metastases from the colon to the liver. Regardless, these same principles of genetic engineering have been applied to a generation of newer constructs.

The recent identification of the neurovirulence gene $\gamma_{1} 34.5$ provided an important target for genetic engineering (5). An HSV-1 variant lacking this gene is more attenuated than the R7020 mutant. In contrast, the corresponding HSV-2 variant appears to be far more virulent, necessitating the deletion of additional genes - which, unfortunately, impairs its ability to replicate to levels required for commercial production. An additional problem is the isolation of second-site compensatory mutants that regain some but not all of the virulence of wild-type strains.

\section{Alternative approaches}

Two other approaches to live-virus HSV vaccines are worthy of brief note. First, the so-called DISC vaccines are replication-defective. One version lacks the essential gene $g H$ and can therefore undergo only one round of replication. Alternative DISC vaccines have been generated by deletion of either the ICP27 or the ICP8 gene (39, 40). Although these candidate viruses are unable to spread, they should generate a broad range of immune responses, and indeed, these vaccines have proved immunogenic, stimulating both humoral and cellmediated immune responses in both mice and guinea pigs (4). A second approach of note is the use of naked DNA, inoculated directly into muscle. The advantage of such an approach is that it allows for both MHC class I and II presentation, perhaps resulting in broader immune responses. The genes attracting the most interest for this application encode ICP27 and HSV-2 gD-2.

\section{Gene therapy applications of replication- attenuated HSV}

Genetically engineered HSV has mainly been assessed for the treatment of human glioblastoma multiforme. These constructs have included mutations in the viral genes for thymidine kinase, ribonucleotide reductase, and $\gamma_{1} 34.5$ (41-43), all of which are intended to improve the therapeutic index in the treatment of gliomas. While virtually any alteration of HSV ameliorates neurovirulence, only the deletions in the $\gamma_{1} 34.5$ gene consistently demonstrate safety and efficacy in animal models. Numerous glioma models, including mouse, rat, and human glioma cell lines and human glioma explants, have been used to demonstrate the tumoricidal effects of these agents in vitro and in vivo - notably increased survival and some tumor cures in intracranial implant models. These effects are reproducible in vivo in both immunodeficient and immunocompetent animals (41, 43-45). The time course of infection indicates impaired replication, with limited spread of virus to the brain (46). The use of two selected mutations, affecting $\gamma_{1} 34.5$ and ribonucleotide reductase, appears to avoid secondsite mutations with reversion to wild-type phenotype (47). Furthermore, the retention of the native HSV TK allows for acyclovir susceptibility in the engineered strain (43). The safety of these constructs has been established in susceptible primates (47).

HSV can also be used as a vector to deliver therapeutic gene products to tumors (refs. 45,48 ; for review 
see ref. 49). Indeed the HSV construct G207 (41) demonstrates an adequate safety profile in both cell culture and animal studies (50) and has proved efficacious in several tumor models in vivo (41-43). This candidate therapeutic is deleted in both copies of the $\gamma_{1} 34.5$ gene as well as in the gene for ribonucleotide reductase. Numerous other constructs have been developed, encoding cytokines and chemokines, enzymes, and receptors (51).

G207, a conditionally replicating HSV mutant, has been evaluated in a phase I safety trial for a subset of patients with unresponsive recurrent malignant gliomas (52). A total of 21 patients receiving escalating doses of G207 at five intratumoral sites showed no toxicity or adverse events that could be unequivocally ascribed to G207 administration, although adverse events were noted in several patients. Importantly, no patient developed herpes simplex encephalitis. Of five seronegative volunteers, two have survived more than 3 years with stable Karnofsky scores. These data provide the basis for phase IB and II clinical trials, recently approved by the Food and Drug Administration, for further dose escalation after tumor debridement or administration of concomitant radiotherapy.

The mutant HSV strain 1716, which is deleted in both copies of the $\gamma_{1} 34.5$ gene and expresses lac $Z$ under the control of the latency-associated transcript, has been studied in Scotland in a similar population. In this trial, a total of nine patients were evaluated at one of three doses of virus, beginning at $1 \times 10^{3}$ and escalating by a factor of 10 to $1 \times 10^{5}(53)$. Again, there were no reports of significant adverse events directly attributable to virus administration. Four of the nine patients were alive 14-24 months after injection. Of note, the maximum amount of virus administered in this trial was four logs lower than that in the study performed in the US (52). These promising studies have led to phase II trials in both the US and the United Kingdom.

Although the two trials examined different genetically engineered constructs and doses of virus for administration, the remarkable demonstration of safety following intratumoral inoculation paves the way for the evaluation of genetically engineered HSV in phase II trials. Future studies are required to address the extent and magnitude of viral replication in the tumor and to characterize the host response in much more detail. Secondgeneration constructs that express the cytokine IL-12 are expected to induce an enhanced effect within the tumor bed and will be used in human investigations in the immediate future. Furthermore, a construct identical to R7020, called NV1020, is under investigation for the selective treatment of colorectal metastases to the liver. In a dose escalation study, this virus has been administered at up to $3 \times 10^{7} \mathrm{PFU}$ without adverse events. Such studies not only lay the groundwork for the broader use of HSV and related vectors in cancer therapy but may well offer important lessons for the development of vaccines to prevent HSV infections.

\section{Conclusion}

Several creative potential prophylactic vaccine candidates have emerged and undergone extensive testing.
The results in humans are mixed, but a hint of efficacy exists. We have learned, for example, that seronegative individuals at high risk for infection represent ideal candidates for participation in vaccine trials, while individuals with frequent recurrences are not significantly affected by vaccines. As a consequence, vaccination should be scheduled for a time prior to exposure of the offending pathogen. For a vaccine designed to prevent HSV-2 infections, this would be early in adolescence prior to the onset of sexual activity.

Adequate methodology has not uniformly been applied to clinical evaluations of HSV vaccines. Current and future studies, to benefit from this experience, should be double-blind and placebo-controlled and should involve a sufficient number of volunteers to provide adequate power for the necessary statistical analyses. Interim analyses, predicated on results obtained during the performance of the trial, will guarantee the ethical nature of the trial design. The value or failure of the current, seemingly promising, approaches to engineering HSV vaccines should be evident within the next several years.

\section{Acknowledgments}

Studies performed by the authors and reported herein were initiated and supported under a contract (NO1AI-65306, NO1-AI-15113, NO1-AI-62554) with the Development and Applications Branch of the National Institute of Allergy and Infectious Diseases, a Program Project Grant, University of Alabama at Birmingham (PO1-AI-24009) (B. Roizman), and grants from the General Clinical Research Center Program (RR032) (R. Whitley), the State of Alabama (R. Whitley), and the National Cancer Institute (CA-78766, CA71933, CA-83939, CA-88860) (B. Roizman).

1. Roizman, B., and Pellett, P.E. 2001. Herpesviridae. In Fields virology. 4th edition. D.M. Knipe and R.M. Howley, editors. Lippincott Williams \& Wilkins. Philadelphia, Pennsylvania, USA. 2381-2397.

2. Roizman, B., and Knipe, D.M. 2001. Herpes simplex viruses and their replication. In Fields virology. 4th edition. D.M. Knipe and R.M. Howley, editors. Lippincott Williams \& Wilkins. Philadelphia, Pennsylvania, USA. 2399-2459.

3. Ward, P.L., and Roizman, B. 1994. Herpes simplex genes: the blueprint of a successful human pathogen. Trends Genet. 10:267-274.

4. Boursnell, M.E., et al. 1997. A genetically inactivated herpes simplex virus type 2 (HSV-2) vaccine provides effective protection against primary and recurrent HSV-2 disease. J. Infect. Dis. 175:16-25.

5. Chou, J., Kern, E.R., Whitley, R.J., and Roizman, B. 1990. Mapping of herpes simplex virus-1 neurovirulence to gamma 134.5, a gene nonessential for growth in culture. Science. 250:1262-1266.

6. Whitley, R.J. 2002. Herpes simplex vaccines. In New generation vaccines. M.M. Levine, J.B. Kaper, R. Rappuoli, M. Liu, and M. Good, editors. Marcel Dekker Inc. New York, New York, USA. In press.

7. Meignier, B., Martin, B., Whitley, R., and Roizman, B. 1990. In vivo behavior of genetically engineered herpes simplex viruses R7017 and R7020. II. Studies in immunocompetent and immunosuppressed owl monkeys (Aotus trivirgatus). J. Infect. Dis. 162:313-321.

8. Lazar, M.P. 1956. Vaccination for recurrent herpes simplex infection: initiation of a new disease site following the use of unmodified material containing the live virus. Arch. Dermatol. 73:70.

9. Jawetz, E., Allende, M.E., and Coleman, V.R. 1955. Studies on herpes simplex virus. VI. Observations on patients with recurrent herpetic lesions injected with herpes viruses or their antigens. Am. J. Med. Sci. 229:477-485

10. Kern, A.B., and Schiff, B.L. 1964. Vaccine therapy in recurrent herpes simplex. Arch. Dermatol. 89:844-845.

11. Chapin, H.B., Wong, S.C., and Reapsome, J. 1962. The value of tissue culture vaccine in the prophylaxis of recurrent attacks of herpetic keratitis. Am. J. Ophthalmol. 54:255-265.

12. Nasemann, T., and Schaeg, G. 1973. Herpes simplex virus, type II: micro- 
biology and clinical experiences with attenuated vaccine. Hautarzt. 24:133-139.

13. Dundarov, S., Andonov, P., Bakalov, B., Nechev, K., and Tomov, C. 1982 Immunotherapy with inactivated polyvalent herpes vaccines. Dev. Biol. Stand. 52:351-358.

14. Soltz-Szots, J. 1971. Therapy of recurrent Herpes simplex. Z. Haut. Geschlechtskr. 46:267-272.

15. Anderson, S.G., Hamilton J., and Williams, S. 1950. An attempt to vaccinate against herpes simplex. Aust. J. Exp. Biol. Med. Sci. 28:579-584.

16. Mertz, G.J., et al. 1984. Herpes simplex virus type-2 glycoprotein-subunit vaccine: tolerance and humoral and cellular responses in humans. J. Infect. Dis. 150:242-249.

17. Cappel, R., DeCuyper, F., and Rikaert, F. 1980. Efficacy of a nucleic acid free herpetic subunit vaccine. Arch. Virol. 65:15-23.

18. Kitces, E.N., Morahan, P.O., Tew, J.G., and Murray, B.K. 1977. Protection from oral herpes simplex virus infection by a nucleic acid-free virus vaccine. Infect. Immun. 16:955-960.

19. Dix, R.D., and Mills, J. 1985. Acute and latent herpes simplex virus neurological disease in mice immunized with purified virus-specific glycoproteins $\mathrm{gB}$ or $\mathrm{gD}$. J. Med. Virol. 17:9-18.

20. Lasky, L.A., Dowbenko, D., Simonsen, C.C., and Berman, P.W. 1984. Protection of mice from lethal herpes-simplex virus-infection by vaccination with a secreted form of cloned glycoprotein-D. Bio-Technology. 2:527.

21. Meignier, B., Jourdier, T.M., Norrild, B., Pereira, L., and Roizman, B 1987. Immunization of experimental animals with reconstituted glycoprotein mixtures of herpes simplex virus 1 and 2: protection against challenge with virulent virus. J. Infect. Dis. 155:921-930.

22. Stanberry, L.R., Bernstein, D.I., Burke, R.L., Pachl, C., and Myers, M.G. 1987. Vaccination with recombinant herpes simplex virus glycoproteins: protection against initial and recurrent genital herpes. J. Infect. Dis. 155:914-920.

23. Stanberry, L.R., Myers, M.G., Stephanopoulos, D.E., and Burke, R.L. 1989. Preinfection prophylaxis with herpes simplex virus glycoprotein immunogens: factors influencing efficacy. J. Gen. Virol. 70:3177-3185.

24. Wachsman, M., et al. 1987. Protection of guinea pigs from primary and recurrent herpes simplex virus (HSV) type 2 cutaneous disease with vaccinia virus recombinants expressing HSV glycoprotein D. J. Infect. Dis. 155:1188-1197.

25. Mertz, G.J., et al. 1990. Double-blind, placebo-controlled trial of a herpes simplex virus type 2 glycoprotein vaccine in persons at high risk for a genital herpes infection. J. Infect. Dis. 161:653-660.

26. Burke, R.L., et al. 1989. Development of herpes simplex virus subunit vaccine. In Vaccines 89: modern approaches to new vaccines including preven tion of AIDS. R.A. Lerner, H. Ginsberg, R.M. Chanock, and F. Brown, editors. Cold Spring Harbor Laboratory Press. Cold Spring Harbor, New York, USA. 377-382.

27. Kaerner, H.C Schroder, C.H., Ott-Hartmann, A., Kummel, G and Kirchner, H. 1983. Genetic variability of herpes simplex virus: development of a pathogenic variant during passaging of a nonpathogenic herpes simplex virus type 1 virus strain in mouse brain. J. Virol. 46:83-93.

28. Straus, S.E., et al. 1994. Placebo-controlled trial of vaccination with recombinant glycoprotein $D$ of herpes simplex virus type 2 for immunotherapy of genital herpes. Lancet. 343:1460-1463.

29. Spruance, S.L., et al. 2000. Gender-specific efficacy of a prophylactic SBAS4-adjuvanted gD2 subunit vaccine against genital herpes disease (GHD): results of two clinical efficacy trials. SmithKline Beecham (SB) Herpes Vaccine Efficacy Study Group. Paper presented at: 40th Interscience Conference on Antimicrobial Agents and Chemotherapy; September 17-20, 2000; Toronto, Canada.

30. Smith, G.L., Mackett, M., and Moss, B. 1983. Infectious vaccinia virus recombinants that express hepatitis B virus surface antigen. Nature. 302:490-495.

31. Paoletti, E., Lipinskas, B.R., Samsonoff, C., Mercer, S., and Panicali, D. 1984. Construction of live vaccines using genetically engineered poxviruses: biological activity of vaccinia virus recombinants expressing the hepatitis B virus surface antigen and the herpes simplex virus glycoprotein D. Proc. Natl. Acad. Sci. USA. 81:193-197.

32. Cremer, K.J., Mackett, M., Wohlenberg, C., Notkins, A.L., and Moss, B.
1985. Vaccinia virus recombinant expressing herpes simplex virus type 1 glycoprotein D prevents latent herpes in mice. Science. 228:737-740.

33. Cantin, E.M., et al. 1987. Expression of herpes simplex virus 1 glycoprotein $B$ by a recombinant vaccinia virus and protection of mice against lethal herpes simplex virus infection. Proc. Natl. Acad. Sci. USA 84:5908-5912.

34. McDermott, M.R., Graham, F.L., Hanke, T., and Johnson, D.C. 1989 Protection of mice against lethal challenge with herpes simplex virus by vaccination with an adenovirus vector expressing HSV glycoprotein B. Virology. 169:244-247.

35. Post, L.E., and Roizman, B. 1981. A generalized technique for deletion of specific genes in large genomes: alpha gene 22 of herpes simplex virus 1 is not essential for growth. Cell. 25:227-232.

36. Mocarski, E.S., Post, L.E., and Roizman, B. 1980. Molecular engineering of the herpes simplex virus genome: insertion of a second L-S junction into the genome causes additional genome inversions. Cell. 22:243-255.

37. Meignier, B., Longnecker, R., and Roizman, B. 1988. In vivo behavior of genetically engineered herpes simplex virus R7017 and R7020. Construction and evaluation in rodents. J. Infect. Dis. 158:602-614.

38. Cadoz, M., et al. Phase 1 trial of R7020: a live attenuated recombinant herpes simplex (HSV) candidate vaccine. Paper presented at: 32 nd Interscience Conference on Antimicrobial Agents and Chemotherapy; October 11-14, 1992; Anaheim, California, USA

39. Da Costa, X.J., Jones, C.A., and Knipe, D.M. 1999. Immunization against genital herpes with a vaccine virus that has defects in productive and latent infection. Proc. Natl. Acad. Sci. USA. 96:6994-6998.

40. de Bruyn Kops, A., Uprichard, S.L., Chen, M., and Knipe, D.M. 1998. Comparison of the intranuclear distributions of herpes simplex virus proteins involved in various viral functions. Virology. 252:162-178.

41. Martuza, R.L., Malick, A., Markert, J.M., Ruffner, K.L., and Coen, D.M. 1991. Experimental therapy of human glioma by means of a genetically engineered virus mutant. Science. 252:854-856.

42. Boviatsis, E.J., et al. 1994. Antitumor activity and reporter gene transfer into rat brain neoplasms inoculated with herpes simplex virus vectors defective in thymidine kinase or ribonucleotide reductase. Gene Ther. 1:323-331.

43. Chambers, R., et al. 1995. Comparison of genetically engineered herpes simplex viruses for the treatment of brain tumors in a scid mouse model of human malignant glioma. Proc. Natl. Acad. Sci. USA. 92:1411-1425.

44. Mineta, T., et al. 1994. CNS tumor therapy by attenuated herpes simplex viruses. Gene Ther. 1(Suppl. 1):S78.

45. Parker, J., et al. 2000. Engineered herpes simplex virus expressing interleukin 12 in the treatment of experimental murine tumors. Proc. Natl. Acad. Sci. USA. 97:2208-2213.

46. Boviatsis, E.J., et al. 1994. Gene transfer into experimental brain tumors mediated by adenovirus, herpes simplex virus, and retrovirus vectors. Hum. Gene Ther. 5:183-191.

47. Yazaki, T., Manz, H.J., Rabkin, S.D., and Martuza, R.L. 1995. Treatment of human malignant meningiomas by G207, a replication-competent multimutated herpes simplex virus 1. Cancer Res. 55:4752-4756.

48. Andreansky, S., et al. 1998. Treatment of intracranial gliomas in immunocompetent mice using herpes simplex viruses that express murine interleukins. Gene Ther. 5:121-130.

49. Chung, S.-M., et al. 2002. The use of a genetically engineered herpes simplex virus (R7020) with ionizing radiation for experimental hepatoma. Gene Ther. 9:75-80.

50. Hunter, W.D., et al. 1999. Attenuated, replication-competent herpes simplex virus type 1 mutant G207: safety evaluation of intracerebral injection in nonhuman primates. J. Virol. 73:6319-6326.

51. Markert, J.M., Gillespie, Y.G., Weichselbaum, R.R., Roizman, B., and Whitley, R.J. 2000. Genetically engineered HSV in the treatment of glioma: a review. Rev. Med. Virol. 10:17-30.

52. Markert, J.M., et al. 2000. Conditionally replicating herpes simplex virus mutant G207 for the treatment of malignant glioma: results of a phase I trial. Gene Ther. 7:867-874.

53. Rampling, R., et al. 2000. Toxicity evaluation of replication-competent herpes simplex virus (ICP 34.5 null mutant 1716) in patients with recurrent malignant glioma. Gene Ther. 7:859-866 